The mean 5 times sit to stand time of 16 seconds was significantly higher than community normals ( $p$ 0.04), demonstrating muscle fatiguability, not isolated to patients with myopathy.

Conclusion OSA is common in patients with PMD, although mostly mild in severity. ESS likely remains a relevant tool in screening patients when considering overnight polysomnography to investigate fatigue in these patients. OSA does not explain the high prevalence of fatigue, with other factors including depressive symptoms, important considerations.

\section{INCIDENCE OF DEMENTIA IN ELDERLY PATIENTS WITH EPILEPSY}

${ }^{1}$ Viral Upadhyay*, ${ }^{2}$ Aloysius Ng, ${ }^{1}$ Manori Wijayath. ${ }^{1}$ Department of Neurology, Nepean Hospital, Sydney, NSW, Australia; ${ }^{2}$ Nepean Clinical School, University of Sydney, Sydney, NSW, Australia

\subsection{6/jnnp-2019-anzan.112}

Introduction The incidence of epilepsy is higher in patients with underlying dementia. ${ }^{1}$ The goal of the present study is to look at the incidence of dementia in patients presented to our epilepsy clinic and analyse electroencephalogram (EEG), imaging findings and response to antiepileptic drug (AED) in these individuals.

Methods A retrospective study was performed on patients presented to Nepean Hospital epilepsy clinic from 2015 to 2017. Multiple clinical parameters were obtained from electronic medical records.

Results A total of 258 patients presented to the clinic, of which 38 patients were above the age of 65 years. 11 patients were excluded due to insufficient information or patients without any history of seizures. Out of the remaining 27 patients studied, nine patients (33\%) had dementia including five patients (19\%) with Alzheimer's dementia. Sixteen patients (59\%) experienced complex partial seizures. Brain MRI was performed in twenty one patients (78\%). Sixteen patients (59\%) had MRI-identified structural lesions including prior stroke or intracerebral haemorrhage. EEGs were performed in twenty patients (74\%). Eight patients (40\%) had abnormal EEG with one patient (5\%) having epileptiform discharges, three patients (15\%) having focal slowing and four patients (20\%) having generalised slowing. Overall, nineteen patients (70\%) were on AEDs with good control and four patients $(15 \%)$ required more than one AED to achieve seizure control.

Conclusion The study showed that there is higher incidence of dementia in patients with epilepsy compared with general population. ${ }^{2}$ Most patients experience complex partial seizures and can be adequately controlled on single AED.

\section{REFERENCES}

1. Rao $S$, Dove $G$, Cascino G, Petersen R. Recurrent seizures in patients with dementia: Frequency, seizure types, and treatment outcome. Epilepsy \& Behavior 2009, 14(1), pp.118-120.

2. ABS (Australian Bureau of Statistics) 2016, Life Tables, States, Territories and Australia, 2013-2015, cat. no. 3302.0.55.001, ABS, Canberra
128 LATE-ONSET POMPE DISEASE (LOPD) PRESENTING WITH FULMINANT HYPERCAPNIC RESPIRATORY FAILURE

${ }^{1}$ Leon S Edwards*, ${ }^{2}$ Michel Tchan, ${ }^{3}$ Kate M Crossley, ${ }^{4}$ Brendon Yee, ${ }^{5}$ Judith M Spies. ${ }^{1}$ Neurology, Liverpool Hospital, Liverpool, NSW, Australia; ${ }^{2}$ Genetic medicine, Westmead Hospital, Westmead, NSW, Australia; ${ }^{3}$ Neurology, Dubbo base hospital, Dubbo, NSW, Australia; ${ }^{4}$ Respiratory and Sleep Medicine, Royal Prince Alfred Hospital, Camperdown, NSW, Australia; 5 Institute of clinical neurosciences, Royal Prince Alfred Hospital, Camperdown, NSW, Australia

\subsection{6/jnnp-2019-anzan.113}

Introduction We report a case of LOPD with acute-on-chronic respiratory failure.

Case A 57 year-old retired farmer presented with obtundation requiring intubation. He reported a 4 month history of hypophonia, intermittent diplopia, lethargy and orthopnea.

Initial arterial blood gas measurement displayed acute-onchronic hypercapnic respiratory failure $(\mathrm{pH} 7.19, \mathrm{pO} 298$ $\mathrm{mmHg}$, pCO2 $112 \mathrm{mmHg}, \mathrm{HCO} 343 \mathrm{mmol} / \mathrm{L})$. Muscle biopsy was suggestive of LOPD with myofibres demonstrating acid phosphatase and periodic acid-schiff positive vacuoles. Diagnosis was confirmed with low $\alpha$-glucosidase activity on dried blood spot $(0.4 \mathrm{umol} / \mathrm{h} / \mathrm{L})$ and elevated urinary tetrasaccharide level $(5 \mathrm{mmol} / \mathrm{mol}$ creatinine). Mutation analysis of the GAA gene demonstrated two known pathogenic mutations (c. $-32-13 \mathrm{~T}>\mathrm{G}$ and c. $1075+1 \mathrm{G}>\mathrm{T}$ ). With improved ventilation, he was able to be extubated. The only respiratory support on discharge was overnight bilevel positive airway pressure ventilation.

Conclusion LOPD is a rare autosomal recessive metabolic disorder caused by a deficiency in acid $\alpha$-glucosidase. This leads to intra-lysomal accumulation of glycogen in tissues. Particularly in the late form, there is significant phenotypic variability. ${ }^{1}$ Diagnosis remains challenging. Cases have been reported with a range of initial symptoms including stroke, ${ }^{2}$ syncope ${ }^{3}$ and chronic respiratory failure. ${ }^{4}$ Acute on chronic respiratory failure at presentation is rare.

Enzyme replacement therapy has been shown to improve both morbidity and mortality in LOPD. ${ }^{5}$ Earlier treatment is associated with better outcomes. ${ }^{6}$ Prompt recognition of cases is paramount. Unexplained acute-on-chronic respiratory failure should raise the possibility of this condition. In such cases, management of ventilation is vital.

\section{REFERENCES}

1. Chan J, et al. The emerging phenotype of late-onset Pompe disease: A systematic literature review. Mol Genet Metab 2017;120(3):163-172.

2. Hossain MA, et al. A Case of Adult-onset Pompe Disease with Cerebral Stroke and Left Ventricular Hypertrophy. J Stroke Cerebrovasc Dis 2018;27 (11):3046-3052.

3. Walczak-Galezewska $M$, et al, Late-onset Pompe disease in a 54 year-old sportsman with an episode of syncope: a case report. Eur Rev Med Pharmacol Sci 2017:21(16):3665-3667.

4. O'Callaghan C, et al, Adult-onset Pompe disease presenting with insidious hypercapnic respiratory failure. Respirol Case Rep 2016;4(5):e00178.

5. Schoser B, S A, Kanters S, Hamed A, Jansen J, Chan K, Karamouzian M, Toscano A. Survival and long-term outcomes in late-onset Pompe disease following alglucosidase alfa treatment: a systematic review and meta-analysis. I Neurol 2017;264(4):621-630.

6. Chien YH, HW, Lee NC. Pompe disease: early diagnosis and early treatment make a difference. Pediatr Neonatol 2013;54(4):219-227. 